\title{
BMJ Open Dietary and physical activity strategies to prevent type 2 diabetes in South Asian adults: protocol for a systematic review
}

\author{
Mirthe Muilwijk, ${ }^{1}$ Karien Stronks, ${ }^{1}$ Samera Azeem Qureshi, ${ }^{2}$ Erik Beune, ${ }^{1}$ \\ Carlos Celis-Morales, ${ }^{3}$ Jason Gill, ${ }^{3}$ Aziz Sheikh, ${ }^{4}$ Anne Karen Jenum, ${ }^{5}$ \\ Irene $\mathrm{GM}$ van Valkengoed ${ }^{1}$
}

To cite: Muilwijk M, Stronks K, Qureshi SA, et al. Dietary and physical activity strategies to prevent type 2 diabetes in South Asian adults: protocol for a systematic review. BMJ Open 2017;7:e012783. doi:10.1136/ bmjopen-2016-012783

- Prepublication history and additional material is available. To view these please visit the journal (http://dx.doi.org/ 10.1136/ bmjopen-2016-012783).

Received 23 May 2016 Revised 15 August 2016 Accepted 6 September 2016

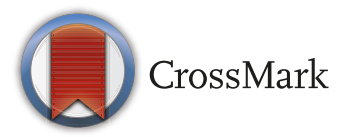

${ }^{1}$ Department of Public Health, Academic Medical Center, University of Amsterdam, Amsterdam, The Netherlands ${ }^{2}$ The Norwegian Centre for Minority Health Research, Oslo, Norway

${ }^{3}$ Institute of Cardiovascular and Medical Sciences, University of Glasgow, Glasgow, UK

${ }^{4}$ Centre for Population Health

Sciences, University of

Edinburgh, Edinburgh, UK

${ }^{5}$ University of Oslo, Oslo, Norway

Correspondence to Ms Mirthe Muilwijk; m. muilwijk@amc.uva.nl, mirthemuilwijk@hotmail.com

\section{ABSTRACT}

Introduction Type 2 diabetes (T2D) is a major health concern among populations of South Asian ethnicity. Although dietary and physical activity interventions may reduce the risk of $\mathrm{T} 2 \mathrm{D}$, the effectiveness has been moderate among South Asians. This might (in part) be because this subgroup follows strategies that were originally developed for interventions among other populations. Therefore, this review aims to assess the evidence for the current dietary and physical activity strategies recommended in T2D prevention intervention studies and guidelines for South Asians.

Methods and analysis Included will be all studies and guidelines on dietary and/or physical activity strategies to prevent T2D in adult South Asians. Two reviewers will search online databases from their start until the present date for published and unpublished experimental/quasiexperimental studies, with at least an abstract in English. References of identified articles and key reviews will be screened for additional studies. Guidelines will be identified by searches in online databases and websites of public organisations. Finally, expert consultations will be held to supplement any missing information. Trial quality will be assessed with the Quality Assessment Tool for Quantitative Studies Data, and guidelines with the Appraisal of Guidelines for Research \& Evaluation II. Data on the strategies recommended, targeting and evidence on effectiveness will be extracted by two reviewers and presented in tabular and narrative forms. Recommendations will be compared with the National Institute for Health and Care Excellence guidelines [PH35]. Overall findings on dietary and physical activity recommendations, as well as findings for specific subgroups (eg, by sex), will be discussed. Ethics and dissemination Ethics assessment is not required. Start date: 1 January 2016, finishing and reporting date 31 July 2016. Results will be published in a peer-reviewed scientific journal, the project report of EuroDHYAN (www.eurodhyan.eu) and in a PhD dissertation. Trial registration number The protocol is registered with the International Prospective Register of Systematic Reviews (PROSPERO) registration number CRD42015027067.
Strengths and limitations of this study

- Our extensive strategy to identify relevant studies and guidelines includes searching a large number of online databases, manually searching public health websites and consultations with relevant experts.

- Quality assessment and reporting of results will be done in accordance with relevant guidelines of systematic reviews.

- Reviewers will not be blinded during quality assessment.

\section{INTRODUCTION}

Populations of South Asian origin are at particularly high risk for type 2 diabetes (T2D). ${ }^{1-3}$ This is of concern because T2D increases the risk of several comorbid conditions including cardiovascular and renal disease, which adversely impacts on quality of life and lifespan. ${ }^{4}$ Diet and physical activity are key modifiable risk factors for T2D. ${ }^{5}$ It is known that interventions with specific strategies targeting these behaviours may prevent or postpone T2D. ${ }^{6-8}$ Strategies for diet and physical activity to prevent T2D have also formed the basis for population-wide prevention guidelines issued by public health organisations. For instance, in the UK, 'eating fibre-rich foods' was included in the T2D prevention 'Population and community-level interventions guideline,' issued by the National Institute for Health and Care Excellence (NICE) ${ }^{9}$

Recently, interventions targeting diet and physical activity have also been implemented to reduce the risk of T2D among populations of South Asian origin. However, the trials evaluating intensive lifestyle interventions in this group show only moderate effects. ${ }^{10-13}$ One interesting observation is that, while the mode of delivery of these 
interventions was often culturally adapted, the recommended dietary and physical activity strategies were often based on those developed for application in other populations. ${ }^{10} 11$ For instance, the intervention implemented in the DHIAAN trial among South Asians in the Netherlands was culturally adapted to that population. ${ }^{10}$ However, the adaptations mainly concerned the design of the intervention and the mode of delivery. ${ }^{14}$ The strategies for diet and physical activity directly followed those originally developed for the general Dutch population. ${ }^{14}$ The use of similar targets may be problematic if the effects of specific changes in physical activity or diet varies across populations; studies suggest that this may be the case. ${ }^{15} 16$ For instance, a difference in the effects of a high-calorie, high-fat diet on insulin sensitivity was found between South Asian and Caucasian men. ${ }^{15}$

This raises the question whether the strategies investigated in intervention trials among South Asians to date are entirely appropriate for South Asian populations. While previous reviews have discussed the effects of interventions on the prevention of T2D, for instance by estimating its effects on weight loss, none has examined the specific strategies that were used. ${ }^{512} 1718$

An evaluation of the different strategies will provide an overview of current strategies and their relevance to the South Asian population which will, ultimately, stimulate the development of more targeted and effective preventive strategies.

Therefore, this systematic review will examine the dietary and physical activity strategies recommended in the guidelines of public organisations and in intervention studies for the prevention of T2D in adult South Asian populations.

The specific questions in this review are:

1. What are the current dietary and physical activity strategies used for the prevention of T2D in adult South Asian populations worldwide?

2. Were the strategies specifically targeted to characteristics of the South Asian population?

3. How effective were the strategies for the prevention of T2D in the South Asian population?

This paper describes the protocol that will be used for the systematic review.

\section{METHODS AND ANALYSIS}

This systematic review protocol is, where appropriate, in line with the preferred reporting items for systematic review/meta-analysis protocols. ${ }^{19}$ The protocol is registered with the International Prospective Register of Systematic Reviews (PROSPERO) registration number CRD42015027067.

\section{Design}

Systematic review.

\section{Eligibility criteria}

We will include intervention studies as well as guidelines. Guidelines are defined as a set of recommended strategies especially developed for the target population. These may include clinical practice guidelines as well as guidelines developed by public organisations, ministries of health, national organisations, primary medical organisations or community-based organisations. Selections of studies as well as guidelines will be based on the following criteria:

\section{Study designs}

- All intervention studies with experimental/ quasiexperimental evaluations will be considered: these include (cluster) randomised controlled trials (RCTs), non-RCTs and before-after studies.

- Modelling studies, study protocols, reviews, retrospective cohort studies and cross-sectional studies will be excluded, as they do not report on experimental evaluations of an intervention in a population.

- All dietary and physical activity guidelines on the prevention of T2D in South Asians will be included.

\section{Participants}

- Only studies or guidelines targeting adults aged $\geq 18$ years will be included.

- The study population should consist of at least $75 \%$ South Asians, or have an identifiable subpopulation of at least $75 \%$ South Asian ethnicity (eg, in a stratified sample). South Asian ethnicity is defined as descending from the South Asian subcontinent, that is, the countries Bangladesh, Bhutan, India, Nepal, Pakistan or Sri Lanka. For studies set in the Indian subcontinent, we will assume that the population is South Asian, unless specified otherwise.

- Studies on pregnant women or malnourished populations will be excluded, as these groups may require specific interventions.

- As the focus of the review is on the prevention of T2D, interventions among populations with T2D are excluded. Studies and guidelines that report on an identifiable subgroup of people without T2D may be considered, for example, in reports combining an investigation of diabetes prevention and management.

\section{Interventions}

- Of interest are dietary and physical activity interventions at the individual, household or community level that aim to reduce T2D risk.

- Studies exclusively on drugs and surgical interventions, or studies on drugs and/or surgical interventions in combination with dietary and/or physical activity interventions will be excluded. However, studies may be included if drugs and surgical interventions are used as alternative interventions or as the comparator 
in a study on dietary and/or physical activity interventions.

\section{Comparators}

All types of comparators will be included, for example, within-group comparisons (before-after study), usual care or alternative interventions, including drugs.

\section{Outcomes of interest}

The outcomes of interest include all descriptive data of dietary and physical activity strategies of the included intervention studies and guidelines. For the description of the effectiveness, the outcomes of interest are related to the effects of the intervention on the prevention of T2D. Therefore, we will consider changes in incident T2D, pre-diabetes and underlying measures of glucose metabolism. Reports on the reduction of overweight/ obesity are also considered if the intervention ultimately aims to reduce the T2D risk.

\section{Primary outcomes are}

- dietary strategies to prevent T2D

- physical activity strategies to prevent T2D.

\section{Secondary outcomes are}

- incidence of T2D

- incidence of pre-diabetes

- indicators of T2D, that is, impaired fasting glucose or impaired glucose tolerance

- anthropometric indicators of adiposity.

The outcomes will be collected as reported, and the measures and definitions used to classify the outcomes will be extracted to facilitate interpretation of the reported findings.

\section{Setting}

All types of settings (eg, individual, household or community level) in which the intervention was carried out will be included. All geographical locations are considered.

\section{Language}

Included will be articles in any language that have an abstract available in English. For full-text papers not available in English, we will translate the methods and results sections. Guidelines written in any language will be included. Manuscripts and guidelines that cannot be translated by a member of the study team will be dealt with on a case-by-case basis.

\section{Time span}

A search will be conducted from the start of each database in our search strategy until the present date. This time limit will cover the relevant period, as previous reviews on interventions in South Asian populations indicate that no studies were conducted before the start of the database. $^{1217}$
Selection based on quality assessment

Studies with a weak rating in the quality assessment will be excluded from the effectiveness assessment. However, studies will be included in the overview of dietary and physical activity strategies regardless of the quality score. This is done because the quality of recommended strategies may be independent from the quality of the conducted study.

\section{Search methods}

\section{Scientific literature}

The search strategy (see online supplementary appendix 1) was developed in collaboration with a clinical librarian from the Academic Medical Center (Amsterdam) and was peer reviewed by members of the project group of the EuroDHYAN study (www.eurodhyan.eu). Eligible published work will be identified by searching for reports in the following databases: PUBMED, Embase, Cochrane Library and Web of Science.

- Unpublished and in-progress studies will be identified by searching the world's largest trial registers, trial registers of the South Asian countries and registers of the top five emigration countries of the South Asian countries with 1 million or more emigrants a year. These trial registers are: WHO International Clinical Trials Registry Platform, International Clinical Trials Registry Platform (ICTRP), ClinicalTrials.gov, ISRCTN Register, EU Clinical Trials Register, the Clinical Trials Register India, the Sri Lanka Clinical Trials Registry, The Saudi Clinical Trials Registry, UK Clinical Trials Gateway and Health Canada's Clinical Trials Database. The search strategy is presented in online supplementary appendix 2 . The study leaders of the identified unpublished and in-progress studies will be contacted to establish whether published literature was missed. We will provide an overview of ongoing studies that have not yet published any results.

Additionally, the reference lists of five key reviews ${ }^{5} 12171820$ and of all the included studies will be screened.

- Expert consultations will be held to identify any missing studies.

- The search strategy is restricted by the time span (start of the database to present) and language (abstract available in English).

\section{Guidelines}

- Two reviewers will electronically search for relevant guidelines in the following databases: the US Guidelines Clearing House, Canadian Medical Association Infobase: Clinical Practice Guidelines Database (CPGs), The Scottish Intercollegiate Guidelines Network, National Health and Medical Research Council: Clinical Practice Guidelines, eGuidelines, International Guideline Library. The search strategy is presented in online supplementary appendix 2. 
- Other dietary and physical activity guidelines will be identified by online searches of the grey literature. We will search for websites of public organisations on the worldwide level, such as the WHO and the Food and Agriculture Organisation. In addition, websites will be searched of ministries of health and nutrition, nutrition centres, physical activity organisations, primary medical organisations for diabetes and community-based organisations operating at the national, state or province level.

- The websites of these organisations will be identified using a publicly available search engine (Google) and visiting web pages of the local government. After identification of the web pages of the various organisations, the individual websites will be searched for the information that is needed by clicking the appropriate headings and/or by use of internal search engines.

- Websites that cannot be translated by any of the team members will be handled on a case-by-case basis.

- These searches will be conducted in all South Asian countries: Bangladesh, Bhutan, India, Nepal, Sri Lanka and Pakistan. In addition, we will include the top five emigration countries of the South Asian countries with 1 million or more emigrants a year: Saudi Arabia, UK, Kuwait, Oman, USA, United Arab Emirates, Canada and Qatar. ${ }^{21}$

- Expert consultations will be held to identify relevant missing guidelines.

\section{Study records}

\section{Data management}

Citation titles and abstracts will be exported from the search engines to Endnote V.X7.4. Duplicates will be removed automatically and, thereafter, manually checked for any missed duplicates. For citations found eligible during screening of titles and abstracts, we will retrieve full texts and import those into Endnote. A PRISMA (Preferred Reporting Items for Systematic Reviews and Meta-Analyses) flow diagram will be manually created during the screening process. Guideline titles and summaries will be screened on the websites of the search engines and on other relevant websites. Full-text guidelines will be exported from the search engines and websites to a folder on the personal computers. The source of the guidelines and the date on which the guideline was retrieved will be stored. Duplicates will be removed manually and eligible full-text guidelines will be stored in a separate folder.

\section{Study selection}

Two reviewers will independently screen titles and abstracts for eligibility. Discrepancies in screening will be resolved by discussion. A third reviewer will be consulted if no consensus is reached. Full texts will be retrieved for the selected articles, and two reviewers will assess whether these meet the inclusion criteria. Discrepancies will be resolved by discussion and, if no consensus is reached, a third reviewer will be consulted for arbitration. The reasons for exclusion of full texts will be noted.

Guidelines will be selected by two reviewers. Discrepancies in selection will be resolved by discussion and, in case no consensus is reached, a third reviewer will be consulted.

\section{Data extraction}

Data will be extracted from the selected papers by two reviewers. Discrepancies will be resolved by consensus, and a third reviewer will be consulted if necessary. In the description of the data, information from records describing the same study ID will be combined. We aim to describe dietary and physical activity recommendations, and the evidence for any targeted advice for the South Asian population.

For identified intervention studies, data will be extracted from the identified reports and also from protocols describing the intervention. Also, a search will be made by study name for protocols published in the medical literature or on study websites. If these are not available, the corresponding authors will be contacted for the intervention protocols. We will register contact attempts and report whether protocols were retrieved. Corresponding authors of the included studies will also be contacted to resolve any ambiguities.

For identified guidelines, the URL and the date of the website visit will be recorded. Data are extracted from the guidelines by two reviewers. Discrepancies are resolved by consensus and, if necessary, a third reviewer is consulted. The primary outcomes are the dietary and physical activity recommendations made. Further, we will examine whether and how recommendations were targeted to the South Asian population, including references that provide evidence for these adaptations.

The following data will be extracted for intervention studies:

1. study ID;

2. reported study type (eg, RCT and before-after study);

3. geographical setting (country and (sub)urban/ rural);

4. setting of the study (eg, household, communitybased or individual);

5. included sample size/number of clusters + cluster sizes;

6. relevant demographic characteristics (including age, ethnicity, country of origin, $\%$ male and sociodemographic characteristics);

7. description of intervention, type of interventions and exact dietary (grouped by nutrients, products or food patterns) and physical activity interventions;

8. intervention strategy;

9. duration of the intervention;

10. comparator;

11. intended and reported study duration;

12. inclusion/exclusion criteria; 
13. primary and secondary aims of the study (eg, weight loss or prevention of T2D);

14. outcomes;

15. effect sizes;

16. confounders adjusted for;

17. bias assessment;

18. follow-up rate and handling of dropouts;

19. follow-up period;

20. adaptations for South Asian target group;

21. evidence used to underpin the strategies included in the interventions.

The following data will be extracted for guidelines:

1. organisation name;

2. geographical setting (country);

3. operating level, for example, national or state/ province level;

4. description of intervention, type of interventions and exact dietary (grouped by nutrients, products or food patterns) and physical activity interventions;

5. specified subgroups or inclusion/exclusion criteria;

6. dietary (grouped by nutrients, products or food patterns) and physical activity recommendations;

7. adaptations for South Asian target group;

8. evidence used to underpin the strategies included in the guidelines.

\section{Risk of bias}

- The Quality Assessment Tool for Quantitative Studies $^{22}$ (developed by the Effective Public Health Practice Project) will be used for a quality assessment of the included studies.

- The reviewers will not be blinded to the studies during the quality assessment process, because it is expected that the reviewers will be familiar with some or all identified studies.

- Two reviewers will individually rate the components, which include: selection bias, study design, confounders, blinding, data collection methods, withdrawals and dropouts, intervention integrity and analyses. Each section can be rated strong, moderate or weak.

- The overall study will be rated according to the section ratings. No weak ratings will result in a strong study rating, while one weak rating will result in a moderate rating for the study, and two or more weak ratings will result in a weak overall rating.

- The quality of guidelines will be assessed by the Appraisal of Guidelines for Research \& Evaluation II instrument.

- Two reviewers will independently rate the components which include: scope and purpose, stakeholder involvement, rigour of development, clarity of presentation, applicability and editorial independence. Items are scored on a 7-point scale.

- For both types of quality assessment methods agreement of reviewers will be checked, in case of disagreement a discussion will be held and, if no consensus is reached, a third reviewer will judge.
- If the study quality is judged to be weak, the study results will not be used to assess the effectiveness of the interventions. However, the dietary and physical activity recommendations and evidence base for these recommendations will still be reported.

\section{Data analysis}

Synthesis

Data on dietary and physical activity recommendations from the intervention studies and from the guidelines will be presented in tabular and narrative forms to provide an overview of the currently used dietary and physical activity strategies to prevent T2D in adult South Asian populations. The results will be compared with the NICE guidelines [PH35] 'Type 2 diabetes prevention: population and community-level interventions,' published in May 2011. ${ }^{9}$ This is the most recent guideline for the prevention of T2D in the general population. Comparing identified strategies with the strategies from the NICE guidelines will enable us to identify to what extent strategies match or deviate from the current insights in strategies that were developed to prevent T2D in the general population. We will identify whether the strategies were specifically targeted to characteristics of the South Asian population, and will describe the available evidence for the effectiveness of these adaptations. This will include reviewing any citations given for evidence on the effectiveness of the recommended changes in relation to the risk of T2D or the glucose metabolism among South Asians. In addition, we will consider any evidence on the effectiveness of (targeted) strategies from the included intervention studies if quality was assessed to be moderate or strong. Finally, we may look for further observational data on the prevalence of the dietary and physical activity strategies and the association of components with T2D in the scientific literature. In addition, in the evaluation of our findings, we will analyse variations in the recommendations by population subgroups. Subgroups can consist of various ethnic groups, migrant/non-migrant groups, geographical setting (Europe/USA/Asia/Oceania), sex (men/women) and/or age ( $\leq 50$ years $/ \geq 50$ years)

\section{AMENDMENTS}

In case of amendments, we will provide the date of each amendment and a description of the change and its rationale in this section. No changes will be incorporated in the protocol. MM will be responsible for approving, documenting and implementing the amendments.

Acknowledgements The authors thank Faridi S van Etten-Jamaludin, clinical librarian at the Academic Medical Center, for her help with the development of the search strategy for the different databases.

Contributors Conceptualisation: MM, KS and IGMV. Methodology: MM, KS, AKJ, AS and IGMV. Writing original draft: MM. Writing review and editing: MM, KS, JG, CCM, AS, SAQ, AKJ, EB and IGMV. Funding acquisition: KS and IGMV. Guarantors: MM and IGMV.

Funding This work was sponsored by the Health Programme 2014-2020 from the European Union, grant number 664609 HP-PJ-2014. 
Competing interests None declared.

Provenance and peer review Not commissioned; externally peer reviewed.

Open Access This is an Open Access article distributed in accordance with the Creative Commons Attribution Non Commercial (CC BY-NC 4.0) license, which permits others to distribute, remix, adapt, build upon this work non-commercially, and license their derivative works on different terms, provided the original work is properly cited and the use is non-commercial. See: http://creativecommons.org/ licenses/by-nc/4.0/

(c) Article author(s) (or their employer(s) unless otherwise stated in the text of the article) 2017. All rights reserved. No commercial use is permitted unless otherwise expressly granted.

\section{REFERENCES}

1. Gujral UP, Pradeepa R, Weber MB, et al. Type 2 diabetes in South Asians: similarities and differences with white caucasian and other populations. Ann N Y Acad Sci 2013;1281:51-63.

2. Meeks KA, Freitas-Da-Silva D, Adeyemo A, et al. Disparities in type 2 diabetes prevalence among ethnic minority groups resident in Europe: a systematic review and meta-analysis. Intern Emerg Med 2016:11:327-40.

3. Sattar N, Gill JM. Type 2 diabetes in migrant south Asians: mechanisms, mitigation, and management. Lancet Diabetes Endocrinol 2015;3:1004-16.

4. IDF. Global guideline for type 2 diabetes: international diabetes federation, 2012.

5. Ramachandran A, Ambady R, Snehalatha C, Samith Shetty A, et al. Primary prevention of type 2 diabetes in South Asians--challenges and the way forward. Diabet Med 2013;30:26-34.

6. Alberti KG, Zimmet P, Shaw J. International Diabetes Federation: a consensus on type 2 diabetes prevention. Diabet Med 2007;24:451-63.

7. Lindström J, Louheranta A, Mannelin M, et al. The Finnish Diabetes Prevention Study (DPS): Lifestyle intervention and 3-year results on diet and Physical activity. Diabetes Care 2003;26:3230-6.

8. The Diabetes Prevention Program (DPP): description of lifestyle intervention. Diabetes Care 2002;25:2165-71.

9. NICE. Type 2 diabetes prevention: population and community-level interventions: national Institute for Health and Clinical Excellence, NICE Guidelines [PH35], 2011. (Last accessed: 23-5-2016).
10. Admiraal WM, Vlaar EM, Nierkens V, et al. Intensive lifestyle intervention in general practice to prevent type 2 diabetes among 18 to 60 -year-old South Asians: 1 -year effects on the weight status and metabolic profile of participants in a randomized controlled trial. PLoS One 2013:8:e68605.

11. Wallia S, Bhopal RS, Douglas A, et al. Culturally adapting the prevention of diabetes and obesity in South Asians (PODOSA) trial. Health Promot Int 2014;29:768-79.

12. Brown T, Smith S, Bhopal R, et al. Diet and physical activity interventions to prevent or treat obesity in south asian children and adults: a systematic review and meta-analysis. Int $J$ Environ Res Public Health 2015;12:566-94.

13. Ramachandran A, Snehalatha C, Mary S, et al. The Indian Diabetes Prevention Programme shows that lifestyle modification and metformin prevent type 2 diabetes in Asian Indian subjects with Impaired glucose tolerance (IDPP-1). Diabetologia 2006;49:289-97.

14. Nicolaou M, Vlaar E, van Valkengoed I, et al. Development of a diabetes prevention program for Surinamese South Asians in the Netherlands. Health Promot Int 2014;29:680-91.

15. Bakker LE, van Schinkel LD, Guigas B, et al. A 5-day high-fat, highcalorie diet impairs insulin sensitivity in healthy, young south asian men but not in caucasian men. Diabetes 2014;63:248-58.

16. Celis-Morales CA, Ghouri N, Bailey ME, et al. Should physical activity recommendations be ethnicity-specific? evidence from a cross-sectional study of south asian and european men. PLoS One 2013;8:e82568.

17. Chapman J, Qureshi N, Kai J. Effectiveness of physical activity and dietary interventions in south asian populations: a systematic review. Br J Gen Pract 2013;63:104-14.

18. Chowdhury TA, Hitman GA. Type 2 diabetes in people of south asian origin: potential strategies for prevention. Br J Diabetes Vasc Dis 2007;7:279-82.

19 Moher D, Shamseer L, Clarke M, et al. PRISMA-P Group. Preferred reporting items for systematic review and meta-analysis protocols (PRISMA-P) 2015 statement. Syst Rev 2015;4:1-349.

20. Balk EM, Earley A, Raman G, et al. Combined diet and physical activity promotion Programs to prevent type 2 Diabetes among persons at increased risk: a systematic review for the Community Preventive services task forcecombined diet and physical activity promotion programs to prevent diabetes. Ann Intern Med 2015;163:437-51.

21. Worldbank. Migration remittances factbook, 2011.

22. Thomas H. Quality assessment tool for quantitative studies Effective Public Health Practice Project. Hamilton, ON, Canada: McMaster University, 2003. 